\title{
Research on the Adaptive Damping Method of Strap-Down Inertial Measurement Unit Based on the Magneto Rheological Technology
}

\author{
Heng Zhang, Xiaoting Rui, Fufeng Yang, and Gangli Chen \\ Institute of Launch Dynamics, Nanjing University of Science \& Technology, Nanjing, Jiangsu 210094, China \\ Correspondence should be addressed to Xiaoting Rui; ruixt@163.net
}

Received 2 December 2016; Revised 30 May 2017; Accepted 3 July 2017; Published 29 August 2017

Academic Editor: Jussi Sopanen

Copyright (c) 2017 Heng Zhang et al. This is an open access article distributed under the Creative Commons Attribution License, which permits unrestricted use, distribution, and reproduction in any medium, provided the original work is properly cited.

To improve the output accuracy of strap-down inertial measurement unit which is in compound dynamic environment of missile which has a high acceleration, strong impact, and high vibration, an adaptive damping method of taking the magneto rheological elastomer as the vibration damper is designed. This method takes the error between the vibration response of the missile and the dynamic response of SIMU as the performance index of optimal control. By optimal control, the stiffness and damping of the magneto rheological elastomer are automatically adjusted, the vibration response of the SIMU is controlled, and the output accuracy of SIMU is improved. Results of experiments show that the method solves the problem that the stiffness and damping of the SIMU damping system could not be changed in real time to adapt to the dynamic environment, and there is almost no time delay and this has an obvious damping effect.

\section{Introduction}

During the launch and flight process of the missile, the mass and stiffness distribution of the missile are changing continuously, and the vibration characteristic of the missile with the flight time is changing continuously. Because the strap-down inertial measurement unit is directly mounted to the missile, the vibration of the missile is transferred to the IMU, which leads to the gyroscopic drift caused by centroid offset and structure deformation of gyroscope, and the dynamic measurement error of the inertia device is generated. When the working frequency of the IMU is close to its resonant frequency, it will greatly reduce the dynamic accuracy of IMU or make permanent damage to the IMU, even output false information, and make the missile completely out of control. Therefore, the dynamic environment adaptability of the strap-down inertial measurement unit is an important subject we have been faced with. In order to improve the dynamic environment adaptability of SIMU, we usually choose the method of testing stiffness or damping of the connection tables or gaskets between the missile and SIMU to reduce the vibration response and improve the dynamic accuracy of inertial navigation system. Because of theoretical and technical reasons, so far, the stiffness and damping of the connection tables or gaskets between the missile and SIMU are constant values, which could not be numerically changed with the change of the vibration frequency of the system [1]. In the flight process of the missile, a large change range of vibration frequency makes it have a different frequency distribution at different time, and the frequency distribution is very dense and wide [2]. Therefore, ordinary damping systems are often difficult to adapt themselves to the dynamic environment of all vibration frequency of flight process of the missile; even they are difficult to avoid the resonance frequency in a certain period of time and improve the accuracy of SIMU too much.

As a new type of intelligent materials, the magneto rheological elastomer has both advantages of magneto rheological fluid and ordinary elastomer, and it overcomes the shortcoming that the iron powder of MRF is easy to settle. Therefore, the magneto rheological elastomer has the characteristics of controllable stiffness and damping under the magnetic field. In this paper, the magneto rheological elastomer that has a variable stiffness and damping is applied 


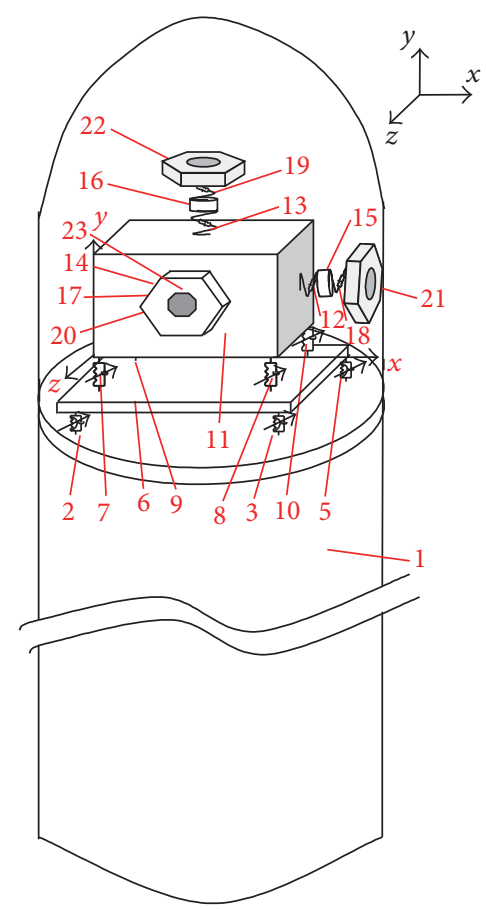

FIGURE 1: The multibody system dynamics model of laser gyro strapdown inertial measurement unit.

to the damping system for SIMU. The magnitude of current of the excitation coil is changed to control the stiffness and damping of the magneto rheological elastomer, thus changing the stiffness and damping of the damping system, to ensure that optimal output control force is produced to eliminate the influence of vibration excitation of the missile at different moments. This method solves the problem that the stiffness and damping of the damping system could not be changed in real time to adapt to the dynamic environment, and this has an obvious effect on the damping system.

\section{The Dynamics Model of Laser Gyro Strap-Down Inertial Measurement Unit and Rapid Calculation of Vibration Characteristics}

2.1. The Multibody System Dynamics Model of Laser Gyro Strap-Down Inertial Measurement Unit. The multibody system dynamics model of laser gyro strap-down inertial measurement unit is shown in Figure 1. The missile is treated as an elastic beam and numbered 1 . The connection between the outer frame and the missile is regarded as a space longitudinal vibration spring and a damper in parallel, numbered 2 to 5. The outer frame of the platform is treated as a rigid body, numbered 6 . The connection between the platform and the outer frame is regarded as a spatial longitudinal vibration spring and a damper in parallel, numbered 7 to 10 . The platform is treated as a rigid body, numbered 11. The connection between the shaking wheel and the platform is regarded as a spatial torsion spring and a damper in parallel, numbered 12,13 , and 14 . The three shaking wheels are treated

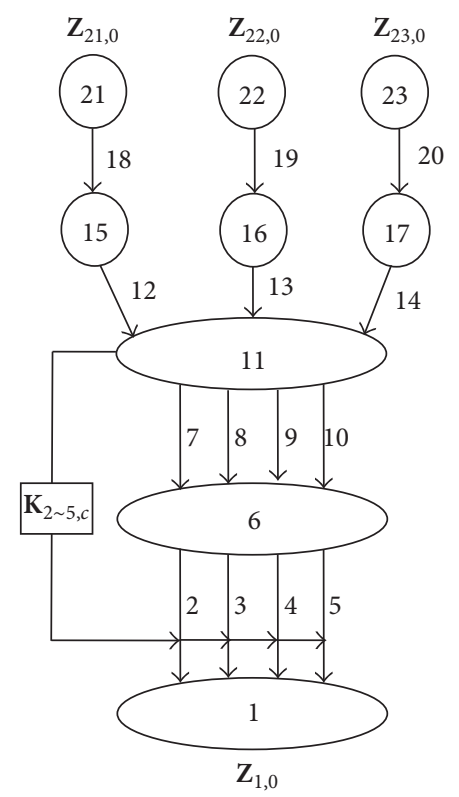

Figure 2: The multibody system topology diagram of laser gyro strap-down inertial measurement unit.

as rigid bodies, numbered 15,16 , and 17 . The connection between the shaking wheel and the gyro cavity is regarded as a spatial torsion spring and a damper in parallel, numbered 18,19 , and 20 . The three gyro cavities are treated as rigid bodies, numbered 21, 22, and 23. Therefore, the multibody system dynamics model of laser gyro strap-down inertial measurement unit is regarded as a multi-rigid-flexible body system which is a combination of eight rigid bodies and one nonuniform elastic beam connected by space springs, torsion springs, and dampers in parallel [3].

The acceleration values of the gyro platform are measured as feedback, then the control forces are applied to element 2 to 5 , and the objective of variable stiffness and damping is achieved. It can be seen from the multibody system dynamics model of laser gyro strap-down inertial measurement unit that the system can be treated as a tree system with three input boundaries and an output boundary. For the convenience of description, the boundary associated with the carrier is treated as the output boundary, and the rest of the boundaries are treated as the input boundaries. The topology diagram of the system is shown in Figure 2.

2.2. The Transfer Equation and Transfer Matrix of Each Element. For the space vibration multibody system, the state vector of the input points, output points, and the system boundary points of each element is defined as [4]

$$
\mathbf{Z}=\left[X, Y, Z, \Theta_{x}, \Theta_{y}, \Theta_{z}, M_{x}, M_{y}, M_{z}, Q_{x}, Q_{y}, Q_{z}\right]^{T}
$$

(a) The transfer equation of the missile with longitudinal vibration, torsional vibration, and transverse vibration is

$$
\mathbf{Z}_{1,0}=\mathbf{U}_{1} \mathbf{Z}_{1, I} .
$$




$$
\mathbf{U}_{1}=\left[\begin{array}{cccccccccccc}
u_{1,1} & 0 & 0 & 0 & 0 & 0 & 0 & 0 & 0 & u_{1,10} & 0 & 0 \\
0 & u_{2,2} & 0 & 0 & 0 & u_{2,6} & 0 & 0 & u_{2,9} & 0 & u_{2,11} & 0 \\
0 & 0 & u_{3,3} & 0 & u_{3,5} & 0 & 0 & u_{3,8} & 0 & 0 & 0 & u_{3,12} \\
0 & 0 & 0 & u_{4,4} & 0 & 0 & u_{4,7} & 0 & 0 & 0 & 0 & 0 \\
0 & 0 & u_{5,3} & 0 & u_{5,5} & 0 & 0 & u_{5,8} & 0 & 0 & 0 & u_{5,12} \\
0 & u_{6,2} & 0 & 0 & 0 & u_{6,6} & 0 & 0 & u_{6,9} & 0 & u_{6,11} & 0 \\
0 & 0 & 0 & u_{7,4} & 0 & 0 & u_{7,7} & 0 & 0 & 0 & 0 & 0 \\
0 & 0 & u_{8,3} & 0 & u_{8,5} & 0 & 0 & u_{8,8} & 0 & 0 & 0 & u_{8,12} \\
0 & u_{9,2} & 0 & 0 & 0 & u_{9,6} & 0 & 0 & u_{9,9} & 0 & u_{9,11} & 0 \\
u_{10,1} & 0 & 0 & 0 & 0 & 0 & 0 & 0 & 0 & u_{10,10} & 0 & 0 \\
0 & u_{11,2} & 0 & 0 & 0 & u_{11,6} & 0 & 0 & u_{11,9} & 0 & u_{11,11} & 0 \\
0 & 0 & u_{12,3} & 0 & u_{12,5} & 0 & 0 & u_{12,8} & 0 & 0 & 0 & u_{12,12}
\end{array}\right],
$$

where

$$
\begin{aligned}
& u_{2,2}=u_{6,6}=u_{9,9}=u_{11,11}=S\left(\lambda_{y} x_{i}\right), \\
& u_{3,3}=u_{5,5}=u_{8,8}=u_{12,12}=S\left(\lambda_{z} x_{i}\right), \\
& u_{2,6}=u_{9,11}=\frac{T\left(\lambda_{y} x_{i}\right)}{\lambda_{y}}, \\
& u_{2,9}=u_{6,11}=\frac{U\left(\lambda_{y} x_{i}\right)}{E I_{z, i} \lambda_{y}^{2}} \\
& u_{2,11}=\frac{V\left(\lambda_{y} x_{i}\right)}{E I_{z, i} \lambda_{y}^{3}} \\
& u_{5,8}=\frac{T\left(\lambda_{z} x_{i}\right)}{E I_{y, i} \lambda_{z}}, \\
& u_{3,5}=u_{8,12}=\frac{-T\left(\lambda_{z} x_{i}\right)}{\lambda_{z}}, \\
& u_{3,8}=u_{5,12}=\frac{-U\left(\lambda_{z} x_{i}\right)}{E I_{y, i} \lambda_{z}^{2}} \\
& u_{3,12}=\frac{V\left(\lambda_{z} x_{i}\right)}{E I_{y, i} \lambda_{z}^{3}} \\
& u_{6,9} \\
& u_{6,3}=\frac{T\left(\lambda_{y} x_{i}\right)}{E I_{z, i} \lambda_{y}} \\
& =u_{12,8}=-\lambda_{z} V\left(\lambda_{z} x_{i}\right) \\
& u_{12,5}=-E I_{y, i} \lambda_{z}^{2} U\left(\lambda_{z} x_{i}\right)
\end{aligned}
$$

$$
u_{8,5}=E I_{y, i} \lambda_{z} V\left(\lambda_{z} x_{i}\right),
$$$$
u_{9,2}=u_{11,6}=E I_{z, i} \lambda_{y}^{2} U\left(\lambda_{y} x_{i}\right) \text {, }
$$$$
u_{9,6}=E I_{z, i} \lambda_{y} V\left(\lambda_{y} x_{i}\right) \text {, }
$$$$
u_{11,2}=E I_{z, i} \lambda_{y}^{3} T\left(\lambda_{y} x_{i}\right) \text {, }
$$$$
u_{12,3}=E I_{y, i} \lambda_{z}^{3} T\left(\lambda_{z} x_{i}\right) \text {, }
$$$$
u_{1,1}=u_{10,10}=\cos \left(\beta_{x} x_{i}\right) \text {, }
$$$$
u_{1,10}=\frac{-\sin \left(\beta_{x} x_{i}\right)}{\beta_{x} E A_{i}} \text {, }
$$$$
u_{10,1}=\beta_{x} E A_{i} \sin \left(\beta_{x} x_{i}\right) \text {, }
$$$$
u_{4,4}=u_{7,7}=\cos \left(\gamma_{\theta_{x}} x_{i}\right) \text {, }
$$$$
u_{4,7}=\frac{\sin \left(\gamma_{\theta_{x}} x_{i}\right)}{\gamma_{\theta_{x}}\left(G J_{p}\right)_{i}} \text {, }
$$$$
u_{7,4}=-\gamma_{\theta_{x}}\left(G J_{p}\right)_{i} \sin \left(\gamma_{\theta_{x}} x_{i}\right) \text {, }
$$$$
0 \leq x_{i} \leq l_{i} \text {, }
$$$$
\beta_{x}=\sqrt{\frac{\overline{m_{i}} \omega^{2}}{E A_{i}}},
$$$$
\lambda_{y}=\sqrt[4]{\frac{\overline{m_{i} \omega^{2}}}{E I_{z, i}}},
$$$$
\lambda_{z}=\sqrt[4]{\frac{\bar{m}_{i} \omega^{2}}{E I_{y, i}}},
$$$$
\gamma_{\theta_{x}}=\sqrt{\frac{\left(\rho J_{p}\right)_{i} \omega^{2}}{\left(G J_{p}\right)_{i}}} .
$$ 
(b) The transfer equation and transfer matrix of elements 2 to 5 and elements 7 to 10 are given as follows.

For dampers 7 10, each damper is treated as a spatial three-direction longitudinal vibration spring, which contains the stiffness of $x, y, z$ direction but does not contain torsional stiffness. The initial stiffness of the four dampers is $\left(k_{x 7}, k_{y 7}, k_{z 7}\right),\left(k_{x 8}, k_{y 8}, k_{z 8}\right),\left(k_{x 9}, k_{y 9}, k_{z 9}\right)$, and $\left(k_{x 10}, k_{y 10}, k_{z 10}\right)$. Since four dampers are clamped between two rigid bodies, the relative displacements of the two bodies should satisfy the rigid body constraints, considering them as a whole, and the input point and output point can be selected at any position of the two rigid bodies. Taking the output point as the original point, the coordinate is established. The coordinates of the balance position of the output end of the four dampers are $\left(a_{7}, b_{7}, c_{7}\right),\left(a_{8}, b_{8}, c_{8}\right),\left(a_{9}, b_{9}, c_{9}\right)$, and $\left(a_{10}, b_{10}, c_{10}\right)$. The coordinates of the balance position of the input end of the four dampers are $\left(a_{7}^{\prime}, b_{7}^{\prime}, c_{7}^{\prime}\right),\left(a_{8}^{\prime}, b_{8}^{\prime}, c_{8}^{\prime}\right)$, $\left(a_{9}^{\prime}, b_{9}^{\prime}, c_{9}^{\prime}\right)$, and $\left(a_{10}^{\prime}, b_{10}^{\prime}, c_{10}^{\prime}\right)$. Assuming that the displacements of the input and output point are $\left[\begin{array}{llllll}x & y & z & \theta_{x} & \theta_{y} & \theta_{z}\end{array}\right]_{I}^{T}$ and $\left[\begin{array}{llllll}x & y & z & \theta_{x} & \theta_{y} & \theta_{z}\end{array}\right]_{O}^{T}$, the space forces generated by each spring damper are simplified to the output point; the principal vectors and principal moments of the force system at the input point and output point are equal. According to this principle, we obtain

$$
\left[\begin{array}{c}
m_{x} \\
m_{y} \\
m_{z} \\
f_{x} \\
f_{y} \\
f_{z}
\end{array}\right]_{O}\left[\begin{array}{c}
m_{x} \\
m_{y} \\
m_{z} \\
f_{x} \\
f_{y} \\
f_{z}
\end{array}\right]_{I}\left[\begin{array}{c}
x \\
y \\
z \\
\theta_{x} \\
\theta_{y} \\
\theta_{z}
\end{array}\right]_{O}-\mathbf{K}_{1}^{\prime}\left[\begin{array}{c}
x \\
y \\
z \\
\theta_{x} \\
\theta_{y} \\
\theta_{z}
\end{array}\right]_{I},
$$

$\left[\begin{array}{c}M_{x} \\ M_{y} \\ M_{z} \\ F_{x} \\ F_{y} \\ F_{z}\end{array}\right]_{O}=\left[\begin{array}{c}M_{x} \\ M_{y} \\ M_{z} \\ F_{x} \\ F_{y} \\ F_{z}\end{array}\right]_{I}\left[\begin{array}{c}X \\ Y \\ Z \\ \Theta_{x} \\ \Theta_{y} \\ \Theta_{z}\end{array}\right]_{O}-\mathbf{K}_{1}^{\prime}\left[\begin{array}{c}X \\ Y \\ Z \\ \Theta_{x} \\ \Theta_{y} \\ \Theta_{z}\end{array}\right]_{I}$,

$$
\left[\begin{array}{c}
X \\
Y \\
Z \\
\Theta_{x} \\
\Theta_{y} \\
\Theta_{z}
\end{array}\right]_{O}=\mathbf{K}_{1}^{-1}\left[\begin{array}{c}
M_{x} \\
M_{y} \\
M_{z} \\
F_{x} \\
F_{y} \\
F_{z}
\end{array}\right]_{I}+\mathbf{K}_{1}^{-1} \mathbf{K}_{1}^{\prime}\left[\begin{array}{c}
X \\
Y \\
Z \\
\Theta_{x} \\
\Theta_{y} \\
\Theta_{z}
\end{array}\right]_{I} .
$$

The transfer equation of elements 7 to 10 is

$$
\mathbf{Z}_{7 \sim 10, O}=\left[\begin{array}{cc}
\mathbf{K}_{1}^{-1} \mathbf{K}_{1}^{\prime} & \mathbf{K}_{1}^{-1} \\
O & I
\end{array}\right]_{7 \sim 10} \mathbf{Z}_{7 \sim 10, I}=\mathbf{U}_{7 \sim 10} \mathbf{Z}_{7 \sim 10, I} .
$$

The transfer matrix of elements 7 to 10 is

$$
\mathbf{U}_{7 \sim 10}=\left[\begin{array}{cc}
\mathbf{K}_{1}^{-1} \mathbf{K}_{1}^{\prime} & \mathbf{K}_{1}^{-1} \\
O & I
\end{array}\right]_{7 \sim 10},
$$

where

$$
\mathbf{K}_{1}=\left[\begin{array}{cccccc}
0 & -\sum_{i=1}^{10} c_{i} k_{y i} & \sum_{i=7}^{10} b_{i} k_{z i} & \sum_{i=7}^{10} k_{y i} c_{i}^{2}+k_{z i} b_{i}^{2} & -\sum_{i=7}^{10} k_{z i} a_{i} b_{i} & -\sum_{i=7}^{10} k_{y i} c_{i} a_{i} \\
\sum_{i=7}^{10} c_{i} k_{x i} & 0 & -\sum_{i=7}^{10} a_{i} k_{z i} & -\sum_{i=7}^{10} k_{z i} a_{i} b_{i} & \sum_{i=7}^{10} k_{x i} c_{i}^{2}+k_{z i} a_{i}^{2} & -\sum_{i=7}^{10} k_{x i} b_{i} c_{i} \\
-\sum_{i=7}^{10} b_{i} k_{x i} & \sum_{i=7}^{10} a_{i} k_{y i} & 0 & -\sum_{i=7}^{10} k_{y i} c_{i} a_{i} & -\sum_{i=7}^{10} k_{x i} b_{i} c_{i} & \sum_{i=7}^{10} k_{x i} b_{i}^{2}+k_{y i} a_{i}^{2} \\
-\sum_{i=7}^{10} k_{x i} & 0 & 0 & 0 & -\sum_{i=7}^{10} k_{x i} c_{i} & \sum_{i=7}^{10} k_{x i} b_{i} \\
0 & -\sum_{i=7}^{10} k_{y i} & 0 & \sum_{i=7}^{10} k_{y i} c_{i} & 0 & -\sum_{i=7}^{10} k_{y i} a_{i} \\
0 & 0 & -\sum_{i=7}^{10} k_{z i} & -\sum_{i=7}^{10} k_{z i} b_{i} & \sum_{i=7}^{10} k_{z i} a_{i} & 0
\end{array}\right] \text {, }
$$




$$
\mathbf{K}_{1}^{\prime}=\left[\begin{array}{cccccc}
0 & -\sum_{i=7}^{10} c_{i}^{\prime} k_{y i} & \sum_{i=7}^{10} b_{i}^{\prime} k_{z i} & \sum_{i=7}^{10} k_{y i} c_{i}^{\prime 2}+k_{z i} b_{i}^{\prime 2} & -\sum_{i=7}^{10} k_{z i} a_{i}^{\prime} b_{i}^{\prime} & -\sum_{i=7}^{10} k_{y i} c_{i}^{\prime} a_{i}^{\prime} \\
\sum_{i=7}^{10} c_{i}^{\prime} k_{x i} & 0 & -\sum_{i=7}^{10} a_{i}^{\prime} k_{z i} & -\sum_{i=7}^{10} k_{z i} a_{i}^{\prime} b_{i} & \sum_{i=7}^{10} k_{x i} c_{i}^{\prime 2}+k_{z i} a_{i}^{\prime 2} & -\sum_{i=7}^{10} k_{x i} b_{i}^{\prime} c_{i}^{\prime} \\
-\sum_{i=7}^{10} b_{i}^{\prime} k_{x i} & \sum_{i=7}^{10} a_{i}^{\prime} k_{y i} & 0 & -\sum_{i=7}^{10} k_{y i} c_{i}^{\prime} a_{i} & -\sum_{i=7}^{10} k_{x i} b_{i}^{\prime} c_{i} & \sum_{i=7}^{10} k_{x i} b_{i}^{\prime 2}+k_{y i} a_{i}^{\prime 2} \\
-\sum_{i=7}^{10} k_{x i} & 0 & 0 & 0 & -\sum_{i=7}^{10} k_{x i} c_{i}^{\prime} & \sum_{i=7}^{10} k_{x i} b_{i}^{\prime} \\
0 & -\sum_{i=7}^{10} k_{y i} & 0 & \sum_{i=7}^{10} k_{y i} c_{i}^{\prime} & 0 & -\sum_{i=7}^{10} k_{y i} a_{i}^{\prime} \\
0 & 0 & -\sum_{i=7}^{10} k_{z i} & -\sum_{i=7}^{10} k_{z i} b_{i}^{\prime} & \sum_{i=7}^{10} k_{z i} a_{i}^{\prime} & 0
\end{array}\right] \text {. }
$$

For dampers 2 5, first considering the situation without control, in the same way, the transfer equation of elements 2 to 5 is

$$
\mathbf{Z}_{2 \sim 5, O}=\left[\begin{array}{cc}
\mathbf{K}_{2}^{-1} \mathbf{K}_{2}^{\prime} & \mathbf{K}_{2}^{-1} \\
O & I
\end{array}\right]_{2 \sim 5} \mathbf{Z}_{2 \sim 5, I}=\mathbf{U}_{2 \sim 5} \mathbf{Z}_{2 \sim 5, I} .
$$

The transfer matrix of elements 2 to 5 is

$$
\mathbf{U}_{2 \sim 5}=\left[\begin{array}{cc}
\mathbf{K}_{2}^{-1} \mathbf{K}_{2}^{\prime} & \mathbf{K}_{2}^{-1} \\
O & I
\end{array}\right]_{2 \sim 5},
$$

where the expressions of $\mathbf{K}_{2}$ and $\mathbf{K}_{2}^{\prime}$ are similar to the expressions of $\mathbf{K}_{1}$ and $\mathbf{K}_{1}^{\prime}$ and $i$ is replaced by 2 to 5 .

Considering the control, that is, adding the accelerometer information of gyro platform to dampers 2 to 5 , the transfer equation of elements 7 to 10 is

$$
\mathbf{Z}_{2 \sim 5, \mathrm{O}}=\mathbf{U}_{2 \sim 5} \mathbf{Z}_{2 \sim 5, I}+\mathbf{U}_{2 \sim 5, c} \mathbf{Z}_{11, I_{1}},
$$

where $\mathbf{U}_{2 \sim 5, c}$ is the matrix related to the position of the accelerometer on the gyro platform and the feedback coefficients of the damper 2 5, point $P$ is the position of the accelerometer on the gyro platform, and $\widetilde{\mathbf{I}}_{I_{1} P}$ is the coordinate matrix of point $P$ relative to the first input point of the gyro platform.

$$
\begin{aligned}
\mathbf{U}_{2 \sim 5, c} & =\left[\begin{array}{cccc}
\mathbf{O}_{3 \times 3} & \mathbf{O}_{3 \times 3} & \mathbf{O}_{3 \times 3} & \mathbf{O}_{3 \times 3} \\
\mathbf{O}_{3 \times 3} & \mathbf{O}_{3 \times 3} & \mathbf{O}_{3 \times 3} & \mathbf{O}_{3 \times 3} \\
\mathbf{K}_{2 \sim 5, c} & -\mathbf{K}_{2 \sim 5, c} \widetilde{\mathbf{l}}_{I_{1} P} & \mathbf{O}_{3 \times 3} & \mathbf{O}_{3 \times 3} \\
\mathbf{O}_{3 \times 3} & \mathbf{O}_{3 \times 3} & \mathbf{O}_{3 \times 3} & \mathbf{O}_{3 \times 3}
\end{array}\right], \\
\mathbf{K}_{2 \sim 5, c} & =\left[\begin{array}{ccc}
k_{2 \sim 5, x d}+i \omega k_{2 \sim 5, x v}-\omega^{2} k_{2 \sim 5, x a} & \\
& k_{2 \sim 5, y d}+i \omega k_{2 \sim 5, y v}-\omega^{2} k_{2 \sim 5, y a} & \\
& k_{2 \sim 5, z d}+i \omega k_{2 \sim 5, z v}-\omega^{2} k_{2 \sim 5, z a}
\end{array}\right] .
\end{aligned}
$$

(c) The transfer equation and transfer matrix of the space elastic hinges are given as follows.

Elements 12, 13, 14, 18, 19, and 20 are space elastic hinges, and the transfer equation is

$$
\mathbf{Z}_{i, \mathrm{O}}=\mathbf{U}_{i} \mathbf{Z}_{i, I} \quad i=12,13,14,18,19,20 .
$$

The transfer matrix is

$$
\mathbf{U}_{i}=\left[\begin{array}{cccc}
\mathbf{I}_{3} & \mathbf{O}_{3 \times 3} & \mathbf{O}_{3 \times 3} & \mathbf{U}_{i, 14} \\
\mathbf{O}_{3 \times 3} & \mathbf{I}_{3} & \mathbf{U}_{i, 23} & \mathbf{O}_{3 \times 3} \\
\mathbf{O}_{3 \times 3} & \mathbf{O}_{3 \times 3} & \mathbf{I}_{3} & \mathbf{O}_{3 \times 3} \\
\mathbf{O}_{3 \times 3} & \mathbf{O}_{3 \times 3} & \mathbf{O}_{3 \times 3} & \mathbf{I}_{3}
\end{array}\right]{ }^{i}=12,13,14,18,19,20,
$$


where

$$
\begin{aligned}
\mathbf{U}_{i, 14} & =\left[\begin{array}{ccc}
-\frac{1}{\mathbf{K}_{i, x}} & 0 & 0 \\
0 & -\frac{1}{\mathbf{K}_{i, y}} & 0 \\
0 & 0 & -\frac{1}{\mathbf{K}_{i, z}}
\end{array}\right], \\
\mathbf{U}_{i, 23} & =\left[\begin{array}{ccc}
\frac{1}{\mathbf{K}_{i, x}^{\prime}} & 0 & 0 \\
0 & \frac{1}{\mathbf{K}_{i, y}^{\prime}} & 0 \\
0 & 0 & \frac{1}{\mathbf{K}_{i, z}^{\prime}}
\end{array}\right],
\end{aligned}
$$

where $\mathbf{K}_{i, x}, \mathbf{K}_{i, y}, \mathbf{K}_{i, z}$ are the stiffness of the $x, y, z$ direction of the longitudinal springs, respectively, and $\mathbf{K}_{i, x}^{\prime}, \mathbf{K}_{i, y}^{\prime}, \mathbf{K}_{i, z}^{\prime}$ are the stiffness of the $x, y, z$ direction of the torsional springs, respectively.

(d) The transfer equation and transfer matrix of the space vibration rigid bodies are shown as follows.

The gyro platform is a space vibration rigid body which has three input ends and one output end, and its transfer equation is

$$
\mathbf{Z}_{11, O}=\mathbf{U}_{11} \mathbf{Z}_{11, I_{1}}+\mathbf{U}_{11, I_{2}} \mathbf{Z}_{11, I_{2}}+\mathbf{U}_{11, I_{3}} \mathbf{Z}_{11, I_{3}}
$$

where

$$
\begin{aligned}
& \mathbf{U}_{11} \\
& =\left[\begin{array}{cccc}
\mathbf{I}_{3} & -\widetilde{\mathbf{l}}_{I_{1} O} & \mathbf{O}_{3 \times 3} & \mathbf{O}_{3 \times 3} \\
O_{3 \times 3} & I_{3} & \mathbf{O}_{3 \times 3} & \mathbf{O}_{3 \times 3} \\
m \omega^{2} \widetilde{\mathbf{l}}_{C O} & -\omega^{2}\left(m \widetilde{\mathbf{l}}_{I_{1} O} \widetilde{\mathbf{l}}_{I_{1} C}+\mathbf{J}_{I_{1}}\right) & \mathbf{I}_{3} & \widetilde{\mathbf{l}}_{I_{1} O} \\
m \mathbf{\Omega}^{2} \mathbf{I}_{3} & -m \omega^{2} \widetilde{\mathbf{l}}_{I_{1} C} & \mathbf{O}_{3 \times 3} & \mathbf{I}_{3}
\end{array}\right], \\
& \mathbf{U}_{11, I_{r}}=\left[\begin{array}{llll}
\mathbf{O}_{3 \times 3} & \mathbf{O}_{3 \times 3} & \mathbf{O}_{3 \times 3} & \mathbf{O}_{3 \times 3} \\
\mathbf{O}_{3 \times 3} & \mathbf{O}_{3 \times 3} & \mathbf{O}_{3 \times 3} & \mathbf{O}_{3 \times 3} \\
\mathbf{O}_{3 \times 3} & \mathbf{O}_{3 \times 3} & \mathbf{I}_{3} & \widetilde{\mathbf{l}}_{I_{r} O} \\
\mathbf{O}_{3 \times 3} & \mathbf{O}_{3 \times 3} & \mathbf{O}_{3 \times 3} & \mathbf{I}_{3}
\end{array}\right]
\end{aligned}
$$

$\mathbf{U}_{11}$ is the transfer matrix of input point $\mathbf{I}_{1}$ to output point $O$ of the rigid body, $\mathbf{U}_{11, I_{r}}(r=2,3)$ is the transfer matrix of input point $\mathbf{I}_{r}(r=2,3)$ to output point $O$ of the rigid body, $\omega$ is the natural frequency of the system, $m$ is the mass, and $\mathbf{J}_{I_{1}}$ is the matrix of moment of inertia of the rigid body relative to the first input point. $\widetilde{\mathbf{l}}_{I_{1} C}$ and $\widetilde{\mathbf{l}}_{I_{1} O}$ are the coordinate matrices of vectors $\overrightarrow{I_{1} C}$ and $\overrightarrow{I_{1} O}$ which are in the body-fixed coordinate system of the rigid body.

For the space vibration rigid body which has several input ends and one output end, only the displacement constraint relationship of the first input point relative to the output point is written into the transfer matrix; the displacement constraint relationships of the other input points relative to the output point are not written though. The geometric constraint equation needs to be added.

$$
\mathbf{H}_{11} \mathbf{Z}_{11, I_{r}}=\mathbf{H}_{11, I_{r}} \mathbf{Z}_{11, I_{1}} \quad(r=2,3),
$$

where

$$
\begin{aligned}
\mathbf{H}_{11, I_{r}} & =\left[\begin{array}{cccc}
\mathbf{I}_{3} & \tilde{\mathbf{I}}_{I_{r} O} & \mathbf{O}_{3 \times 3} & \mathbf{O}_{3 \times 3} \\
\mathbf{O}_{3 \times 3} & \mathbf{I}_{3} & \mathbf{O}_{3 \times 3} & \mathbf{O}_{3 \times 3}
\end{array}\right], \\
\mathbf{H}_{11} & =\left[\begin{array}{cccc}
\mathbf{I}_{3} & \mathbf{O}_{3 \times 3} & \mathbf{O}_{3 \times 3} & \mathbf{O}_{3 \times 3} \\
\mathbf{O}_{3 \times 3} & \mathbf{I}_{3} & \mathbf{O}_{3 \times 3} & \mathbf{O}_{3 \times 3}
\end{array}\right] .
\end{aligned}
$$

Elements $6,15,16,17,21,22$, and 23 are space vibration rigid bodies, and the transfer equation is

$$
\mathbf{Z}_{i, O}=\mathbf{U}_{i} \mathbf{Z}_{i, I} \quad(i=6,15,16,17,21,22,23) .
$$

The transfer matrix is

$$
\begin{aligned}
& \mathbf{U}_{i}=\left[\begin{array}{cccc}
\mathbf{I}_{3} & -\widetilde{\mathbf{l}}_{I_{1} O} & \mathbf{O}_{3 \times 3} & \mathbf{O}_{3 \times 3} \\
\mathbf{O}_{3 \times 3} & \mathbf{I}_{3} & \mathbf{O}_{3 \times 3} & \mathbf{O}_{3 \times 3} \\
m \omega^{2} \widetilde{\mathbf{l}}_{C O} & -\omega^{2}\left(m \widetilde{\mathbf{l}}_{I_{1} O} \widetilde{\mathbf{I}}_{I_{1} C}+\mathbf{J}_{I_{1}}\right) & \mathbf{I}_{3} & \widetilde{\mathbf{l}}_{I_{1} O} \\
m \mathbf{\Omega}^{2} \mathbf{I}_{3} & -m \omega^{2} \widetilde{\mathbf{I}}_{I_{1} C} & \mathbf{O}_{3 \times 3} & \mathbf{I}_{3}
\end{array}\right] \\
& (i=6,15,16,17,21,22,23) \text {. }
\end{aligned}
$$

2.3. The Automatic Derivation of the Transfer Equation Based on the Tree Structure Topology Diagram. The total transfer equation of the system can be obtained by the automatic derivation technique of the transfer equation based on the tree structure topology diagram [5].

$$
\mathbf{Z}_{1,0}=\mathbf{T}_{21-1} \mathbf{Z}_{21,0}+\mathbf{T}_{22-1} \mathbf{Z}_{22,0}+\mathbf{T}_{23-1} \mathbf{Z}_{23,0}
$$

where

$$
\begin{aligned}
& \mathbf{T}_{21-1}=\left(\mathbf{U}_{1} \mathbf{U}_{2 \sim 5} \mathbf{U}_{6} \mathbf{U}_{7 \sim 10}+\mathbf{U}_{1} \mathbf{U}_{2 \sim 5, c}\right) \\
& \cdot \mathbf{U}_{11,12} \mathbf{U}_{12} \mathbf{U}_{15} \mathbf{U}_{18} \mathbf{U}_{21}, \\
& \mathbf{T}_{22-1}=\mathbf{U}_{1} \mathbf{U}_{2 \sim 5} \mathbf{U}_{6} \mathbf{U}_{7 \sim 10} \mathbf{U}_{11,13} \mathbf{U}_{13} \mathbf{U}_{16} \mathbf{U}_{19} \mathbf{U}_{22}, \\
& \mathbf{T}_{23-1}=\mathbf{U}_{1} \mathbf{U}_{2 \sim 5} \mathbf{U}_{6} \mathbf{U}_{7 \sim 10} \mathbf{U}_{11,14} \mathbf{U}_{14} \mathbf{U}_{17} \mathbf{U}_{20} \mathbf{U}_{23} .
\end{aligned}
$$

The constraint equation of the system is

$$
\begin{aligned}
& \mathbf{G}_{21-11} \mathbf{Z}_{21,0}=\mathbf{G}_{22-11} \mathbf{Z}_{22,0}, \\
& \mathbf{G}_{21-11} \mathbf{Z}_{21,0}=\mathbf{G}_{23-11} \mathbf{Z}_{23,0},
\end{aligned}
$$

where $\mathbf{G}_{21-11}=\mathbf{H}_{11,12} \mathbf{U}_{12} \mathbf{U}_{15} \mathbf{U}_{18} \mathbf{U}_{21}, \mathbf{G}_{22-11}=$ $\mathbf{H}_{11,13} \mathbf{U}_{13} \mathbf{U}_{16} \mathbf{U}_{19} \mathbf{U}_{22}$, and $\mathbf{G}_{23-11}=\mathbf{H}_{11,13} \mathbf{U}_{12} \mathbf{U}_{15} \mathbf{U}_{18} \mathbf{U}_{21}$

Equations (22) and (24) are written together and expressed in the form of the matrix

$$
\left[\begin{array}{cccc}
-\mathbf{I} & \mathbf{T}_{21-1} & \mathbf{T}_{22-1} & \mathbf{T}_{23-1} \\
\mathbf{O}_{3 \times 6} & \mathbf{G}_{21-11} & \mathbf{G}_{22-11} & \mathbf{O}_{3 \times 6} \\
\mathbf{O}_{3 \times 6} & \mathbf{G}_{21-11} & \mathbf{O}_{3 \times 6} & \mathbf{G}_{23-11}
\end{array}\right]\left[\begin{array}{c}
\mathbf{Z}_{1,0} \\
\mathbf{Z}_{21,0} \\
\mathbf{Z}_{22,0} \\
\mathbf{Z}_{23,0}
\end{array}\right]=\mathbf{0}
$$


That is,

$$
\mathbf{U}_{\mathrm{all}} \mathbf{Z}_{\mathrm{all}}=\mathbf{0},
$$

where

$$
\begin{aligned}
\mathbf{U}_{\text {all }} & =\left[\begin{array}{cccc}
-\mathbf{I} & \mathbf{T}_{21-1} & \mathbf{T}_{22-1} & \mathbf{T}_{23-1} \\
\mathbf{O}_{3 \times 6} & \mathbf{G}_{21-11} & \mathbf{G}_{22-11} & \mathbf{O}_{3 \times 6} \\
\mathbf{O}_{3 \times 6} & \mathbf{G}_{21-11} & \mathbf{O}_{3 \times 6} & \mathbf{G}_{23-11}
\end{array}\right], \\
\mathbf{Z}_{\text {all }} & =\left[\begin{array}{c}
\mathbf{Z}_{1,0} \\
\mathbf{Z}_{21-0} \\
\mathbf{Z}_{22-0} \\
\mathbf{Z}_{23-0}
\end{array}\right] .
\end{aligned}
$$

Substituting the boundary condition and eliminating the column of zero elements in $\mathbf{U}_{\text {all }}$ and $\mathbf{Z}_{\text {all }}$, we obtain

$$
\overline{\mathbf{U}}_{\mathrm{all}} \overline{\mathbf{Z}}_{\mathrm{all}}=\mathbf{0} \text {. }
$$

2.4. Fast Calculation of Vibration Characteristics of the StrapDown Inertial Measurement Unit. Equation (28) is a homogeneous linear equation set. If it has nonzero solutions, the coefficient determinant is zero, and the characteristic equation of the system is

$$
\operatorname{det}\left(\overline{\mathbf{U}}_{\text {all }}\right)=0 .
$$

The characteristic equation of the system is a transcendental equation with the natural frequency of the system as the unknown number. Dichotomy is used to solve the characteristic root (natural frequency of the system), that is, to utilize the sign change to test whether the characteristic equation has roots in the interval $\left[\omega_{0}, \omega_{0}+\Delta \omega\right]$. If the root exists, dichotomy is used to seek the roots out that satisfy the precision requirement in the interval. If no root exists, continue to check if there is a root in interval $\left[\omega_{0}+\Delta \omega, \omega_{0}+\right.$ $2 \Delta \omega$ ], and so on, until the root is sought out [6]. The solving process of the vibration characteristics of SIMU system is shown in Figure 3. The results of the vibration characteristics of SIMU system using the multibody system transfer matrix method and ordinary dynamics method are compared, as shown in Table 1. It can be seen from Table 1 that multibody system transfer matrix method is 169.9 times the speed of the ordinary dynamics method in the calculation of vibration characteristics of SIMU system.

2.5. The State Space Expression of the System. The body dynamics equation of the system [7] is

$$
\mathbf{M v}_{t t}+\mathbf{K v}=\mathbf{f} .
$$

Applying the modal analysis theory, the response of the system can be expressed by the first $m$ modes

$$
\mathbf{v}=\sum_{k=1}^{m} \mathbf{V}^{k} q^{k}(t)
$$

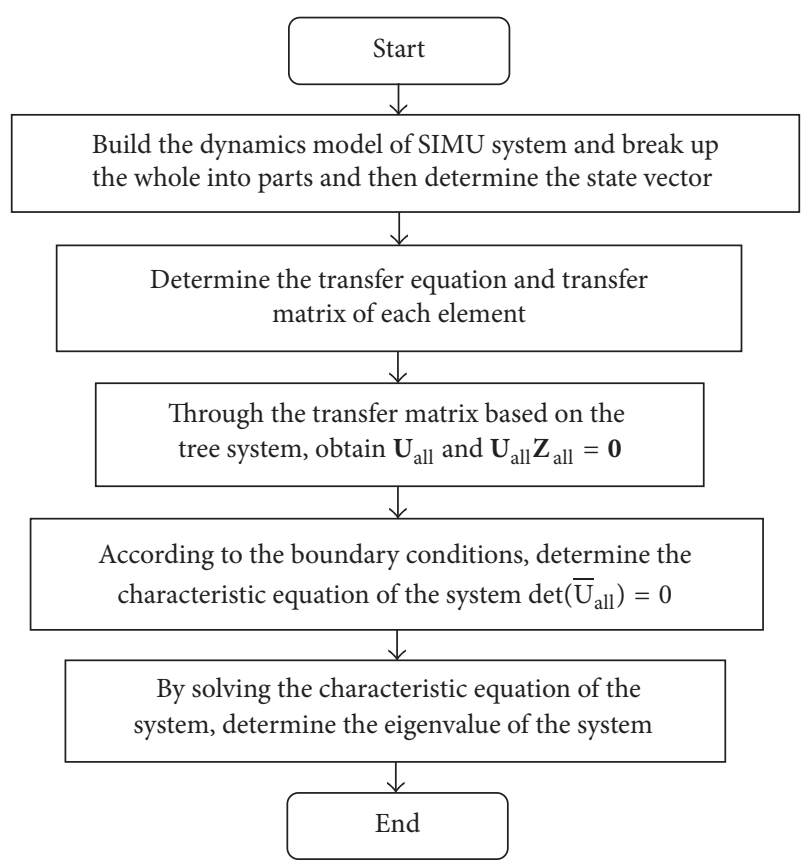

FIGURE 3: The solving process of vibration characteristics of SIMU system.

where $q^{k}(t)$ is the generalized coordinates corresponding to the $k$ th mode of the system and $k$ is the order of the modes. For the vibration system that contains flexible bodies $m=\infty$, the modal truncation is usually used in practical engineering; in other words, the response of the system is approximately expressed as the first $m$ modes when $m$ is chosen big enough. Substituting (31) into (30), then

$$
\sum_{k=1}^{m} \mathbf{M} \mathbf{V}^{k} \ddot{q}^{k}(t)+\sum_{k=1}^{m} \mathbf{K} \mathbf{V}^{k} q^{k}(t)=\mathbf{f} .
$$

Taking inner product [8] of the augmented eigenvector $\mathbf{V}^{p}$ corresponding to the $p$ th mode with both sides of the above equation, then

$$
\begin{aligned}
\sum_{k=1}^{m} & \left\langle\mathbf{V}^{p}, \mathbf{M} \mathbf{V}^{k}\right\rangle \ddot{q}^{k}(t)+\sum_{k=1}^{m}\left\langle\mathbf{V}^{p}, \mathbf{K} \mathbf{V}^{k}\right\rangle q^{k}(t) \\
& =\left\langle\mathbf{V}^{p}, \mathbf{f}\right\rangle .
\end{aligned}
$$

Using orthogonality of augmented eigenvector [9], $m$ independent equations are

$$
\ddot{q}^{k}(t)+\omega_{p}^{2} q^{k}(t)=b^{k} \quad(k=1,2, \ldots, m),
$$

where $b^{k}=\left\langle\mathbf{V}^{k}, \mathbf{f}\right\rangle / \mathbf{M}_{k}$.

Considering the system to be a proportional damping system, assuming $\zeta_{k}$ to be the damping ratio corresponding to the $k$ th mode of the system, the differential equation of the system with damping vibration in modal coordinates is

$$
\ddot{q}^{k}(t)+2 \zeta_{p} \omega^{k} \dot{q}^{k}(t)+\omega_{p}^{2} q^{k}(t)=b^{k},
$$

$$
(k=1,2, \ldots, m) .
$$


TABLE 1: The comparison of multibody system transfer matrix method and ordinary dynamics method.

\begin{tabular}{|c|c|c|c|c|c|c|}
\hline \multirow{2}{*}{$\begin{array}{l}\text { Order of } \\
\text { modes }\end{array}$} & \multicolumn{2}{|c|}{$\begin{array}{l}\text { Multibody system transfer } \\
\text { matrix method }\end{array}$} & \multicolumn{2}{|c|}{ Ordinary dynamic method } & \multicolumn{2}{|c|}{ Comparison } \\
\hline & Frequency/Hz & Time/s & Frequency/Hz & Time/s & Calculation error/\% & Calculation time/times \\
\hline 1 & 18.17 & \multirow{6}{*}{0.775} & 18.18 & \multirow{6}{*}{131.672} & 0.06 & \multirow{6}{*}{169.90} \\
\hline 2 & 37.44 & & 37.43 & & -0.03 & \\
\hline 3 & 48.17 & & 48.15 & & -0.04 & \\
\hline 4 & 48.41 & & 48.45 & & 0.08 & \\
\hline 5 & 62.00 & & 62.05 & & 0.08 & \\
\hline 6 & 93.30 & & 93.35 & & 0.05 & \\
\hline
\end{tabular}

We assume that

According to (36), (38), and (39), we obtain

$$
\mathbf{x}^{k}=\left[\begin{array}{c}
q^{k} \\
\dot{q}^{k}
\end{array}\right]
$$

Equation (35) is written in the form of first-order differential equations; then

$$
\dot{\mathbf{x}}^{k}=\mathbf{A}^{k} \mathbf{x}^{k}+\mathbf{B}^{k} \mathbf{u}, \quad(k=1,2, \ldots, m),
$$

where $\mathbf{A}^{k}=\left[\begin{array}{cc}0 & 1 \\ -\omega_{k}^{2} & -2 \zeta_{k} \omega_{k}\end{array}\right]$ and $\mathbf{B}^{k}=\left[\begin{array}{c}0 \\ b^{k}\end{array}\right]$

Equation (37) is written in the form of matrix

$$
\dot{\mathbf{x}}=\mathbf{A x}+\mathbf{B u},
$$

where

$$
\begin{aligned}
\mathbf{x} & =\left[\begin{array}{c}
\mathbf{x}^{1} \\
\mathbf{x}^{2} \\
\vdots \\
\mathbf{x}^{m}
\end{array}\right], \\
\mathbf{A} & =\operatorname{diag}\left(\begin{array}{llll}
\mathbf{A}^{1} & \mathbf{A}^{2} & \cdots & \mathbf{A}^{m}
\end{array}\right), \\
\mathbf{B} & =\left[\begin{array}{c}
\mathbf{B}^{1} \\
\mathbf{B}^{2} \\
\vdots \\
\mathbf{B}^{m}
\end{array}\right] .
\end{aligned}
$$

Equation (38) is the state equation of the system.

As mentioned above, point $P$ is the position of the accelerometer on the gyro platform; the acceleration of point $P$ is considered to be the output of control, expressed as $\mathbf{y}$.

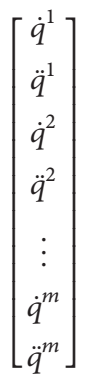

$$
=\left[\begin{array}{ccccccc}
0 & 1 & 0 & 0 & \cdots & 0 & 0 \\
-\omega_{1}^{2} & -2 \zeta_{1} \omega_{1} & 0 & 0 & \cdots & 0 & 0 \\
0 & 0 & 0 & 1 & 0 & \cdots & 0 \\
\vdots & 0 & -\omega_{2}^{2} & -2 \zeta_{2} \omega_{2} & 0 & \vdots & \vdots \\
0 & \cdots & 0 & 0 & \ddots & 0 & 0 \\
0 & 0 & \cdots & 0 & 0 & 0 & 1 \\
0 & 0 & \cdots & 0 & 0 & -\omega_{m}^{2} & -2 \zeta_{m} \omega_{m}
\end{array}\right]\left[\begin{array}{c}
q^{1} \\
\dot{q}^{1} \\
q^{2} \\
\dot{q}^{2} \\
\vdots \\
q^{m} \\
\dot{q}^{m}
\end{array}\right]
$$

$$
+\left[\begin{array}{c}
0 \\
b^{1} \\
0 \\
b^{2} \\
\vdots \\
0 \\
b^{m}
\end{array}\right] \mathbf{u}
$$

Then $\ddot{q}^{m}=-\omega_{m}^{2} q^{m}-2 \zeta_{m} \omega_{m} \dot{q}^{m}+b^{m} \mathbf{u}$.

$$
\begin{array}{r}
\ddot{\mathbf{v}}_{P}=\sum_{k=1}^{m} \mathbf{V}^{k} \ddot{q}_{P}^{k}=\sum_{k=1}^{m} \mathbf{V}^{k}\left(-\omega_{k}^{2} q_{P}^{k}-2 \zeta_{k} \omega_{k} \dot{q}_{P}^{k}+b^{k} \mathbf{u}\right), \\
(k=1,2, \ldots, m),
\end{array}
$$

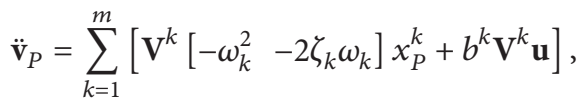

$$
(k=1,2, \ldots, m) .
$$




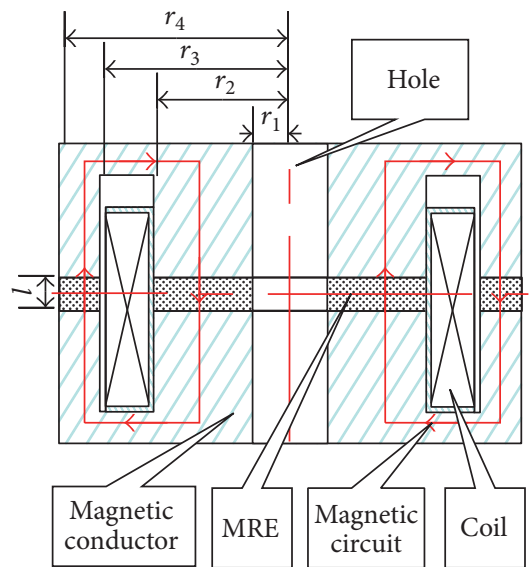

(a) Structure

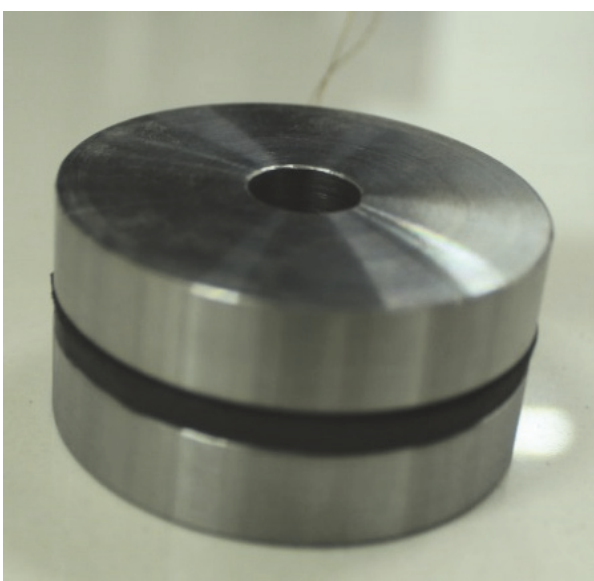

(b) Photograph

FIGURE 4: MRE device.

Then

$$
\mathbf{y}=\ddot{\mathbf{v}}_{P}=\sum_{k=1}^{m}\left[\mathbf{C}^{k} x_{P}^{k}+\mathbf{D}^{k} \mathbf{u}\right], \quad(k=1,2, \ldots, m),
$$

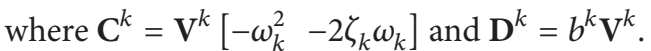

Equation (42) is written in the form of matrix

$$
\mathbf{y}=\mathbf{C x}+\mathbf{D u},
$$

where

$$
\begin{gathered}
\mathbf{x}=\left[\begin{array}{c}
\mathbf{x}^{1} \\
\mathbf{x}^{2} \\
\vdots \\
\mathbf{x}^{m}
\end{array}\right], \\
\mathbf{C}=\left[\begin{array}{c}
\mathbf{C}^{1} \\
\mathbf{C}^{2} \\
\vdots \\
\mathbf{C}^{m}
\end{array}\right], \\
\mathbf{D}=\left[\begin{array}{c}
\mathbf{D}^{1} \\
\mathbf{D}^{2} \\
\vdots \\
\mathbf{D}^{m}
\end{array}\right] .
\end{gathered}
$$

Equation (43) is the output equation of the system, and the state space expression of the system is composed of (38) and (43).

\section{The Mechanical Model of the MRE Device}

The MRE device, which is composed of two MREs, coil and two magnetic conductors, is shown in Figure 4. The size of the MRE device is designed to $r_{1}=2.5 \mathrm{~mm}, r_{2}=14.7 \mathrm{~mm}$, $r_{3}=19.5 \mathrm{~mm}, r_{4}=24.3 \mathrm{~mm}$, and $l=2 \mathrm{~mm}$. According to the size, $r_{2}^{2}-r_{1}^{2}=r_{4}^{2}-r_{3}^{2}$. Therefore, the areas of the two MREs are equal, which can make the magnetic induction intensity of two MREs consistent. So the total area of MREs is $A=$ $\pi\left(r_{2}^{2}-r_{1}^{2}\right)+\pi\left(r_{4}^{2}-r_{3}^{2}\right)=1.3 \times 10^{-3} \mathrm{~m}^{2}$.

The ordinary rubber device which has a similar structure to the MRE device is shown in Figure 5. It is composed of only a piece of rubber and two pieces of metal. The size of the MRE device is designed to $r_{1}^{\prime}=6 \mathrm{~mm}, r_{2}^{\prime}=24 \mathrm{~mm}$, and $l_{r}=8 \mathrm{~mm}$. Therefore, the total area of the rubber is $A_{r}=\pi\left(r_{2}^{\prime 2}-r_{1}^{\prime 2}\right)=$ $1.7 \times 10^{-3} \mathrm{~m}^{2}$.

The middle hole with screw can play the roles of fixed and limited displacement.

The values of tension/compression modulus and loss factor of MRE device with current at loading frequency $(15 \mathrm{~Hz})$ are shown in Figure 6. E represents the complex tension/compressive modulus, which can be expressed

$$
E=E^{\prime}+i E^{\prime \prime}
$$

where $E^{\prime}$ is the storage modulus and $E^{\prime \prime}$ is the loss modulus. The loss factor $\eta$ can be expressed

$$
\eta=\frac{E^{\prime \prime}}{E^{\prime}}
$$

The loss modulus of the ordinary rubber device is $E_{r}^{\prime \prime}=$ $1.33 \mathrm{MPa}$, and the storage modulus of the ordinary rubber device is $E_{r}^{\prime}=3.8 \mathrm{MPa}$. According to (46), $\eta_{r}=E_{r}^{\prime \prime} / E_{r}^{\prime}=0.35$.

$$
\begin{aligned}
& k_{r}=E_{r}^{\prime} \frac{A_{r}}{l_{r}}=8.05 \times 10^{5} \mathrm{~N} / \mathrm{m}, \\
& c_{r}=\frac{\eta_{r} k_{r}}{\omega}=2.99 \times 10^{3} \mathrm{~N} \cdot \mathrm{s} \cdot \mathrm{m}^{-1},
\end{aligned}
$$

where $E_{r}^{\prime}$ is the value of the storage modulus of ordinary rubber, $k_{r}$ is the value of the stiffness of ordinary rubber device, and $c_{r}$ is the value of the damping of ordinary rubber device. 


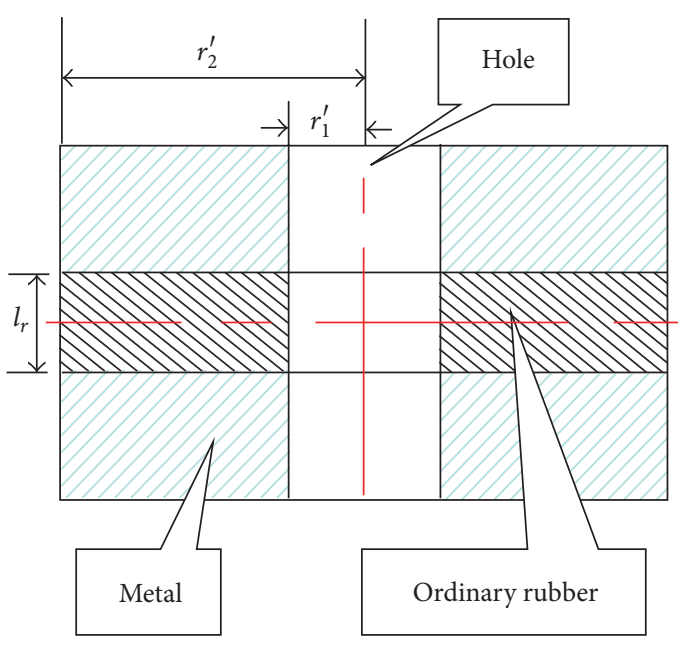

(a) Structure

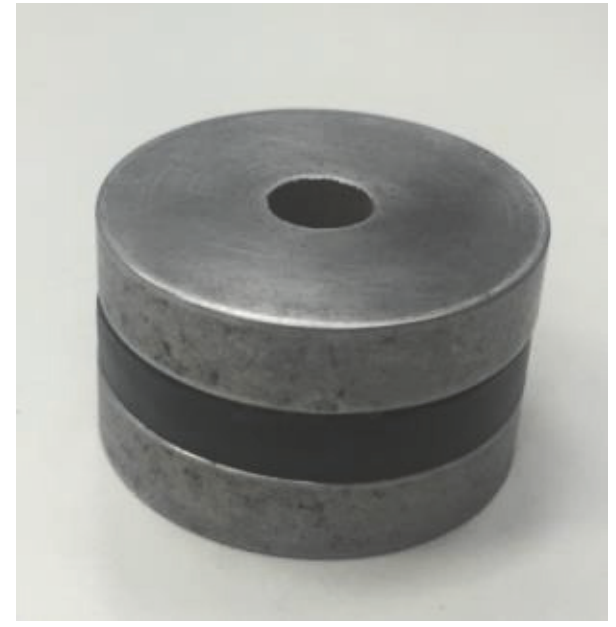

(b) Photograph

Figure 5: The ordinary rubber device.

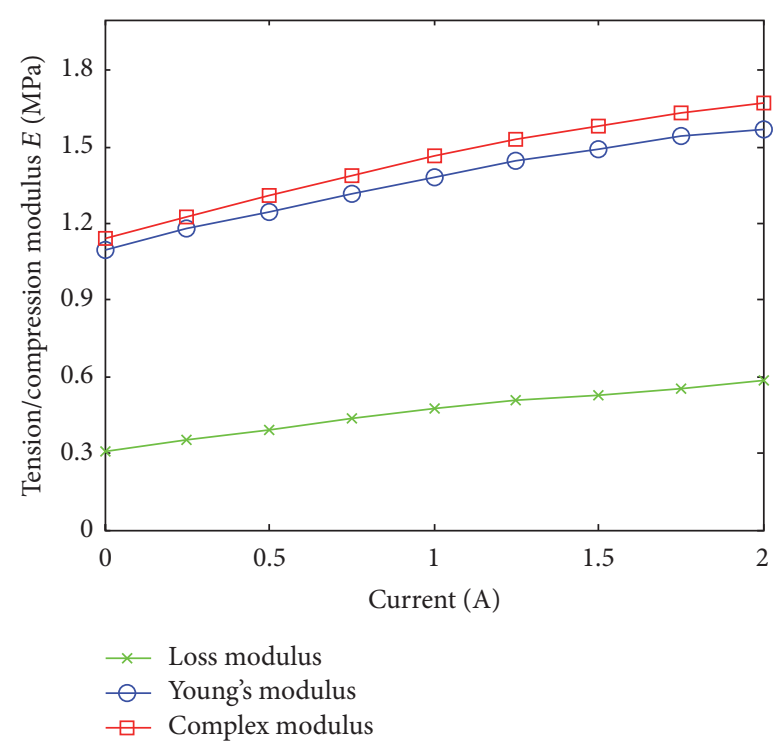

(a) Tension/compression modulus

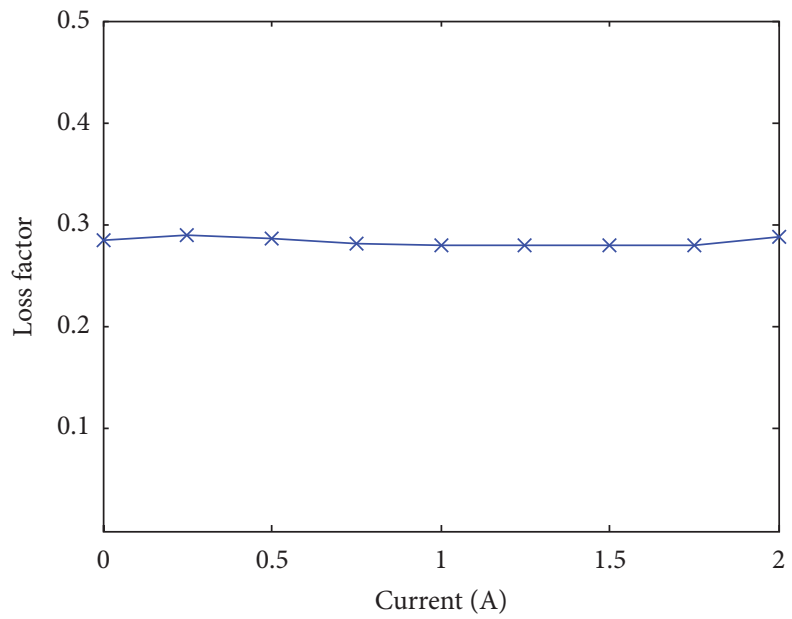

FIGURE 6: Values of the tension/compression modulus and loss factor of MRE device with the current at loading frequency (15 Hz).

From Figure 6, we have

$$
\begin{aligned}
& k_{\min }=E_{\min }^{\prime} \frac{A}{l}=7.13 \times 10^{5} \mathrm{~N} / \mathrm{m}, \\
& k_{\max }=E_{\max }^{\prime} \frac{A}{l}=1.02 \times 10^{6} \mathrm{~N} / \mathrm{m},
\end{aligned}
$$

where $E_{\min }^{\prime}$ and $E_{\max }^{\prime}$ are the minimum and maximum value of the storage modulus of MRE, respectively, and $k_{\min }$ and $k_{\max }$ are the minimum and maximum value of the stiffness of MRE, respectively. by

The stiffness and damping of the MRE device can be given

$$
\begin{aligned}
& k_{\mathrm{MRE}}=k_{\min }+K(i), \\
& c_{\mathrm{MRE}}=\frac{\eta k_{\mathrm{MRE}}}{\omega},
\end{aligned}
$$

where $K(i)$ is a stiffness function of the efficient current $i$. Observing Figure 5, we have

$$
K(i)=-0.0858 i^{2}+0.4877 i-0.0233
$$




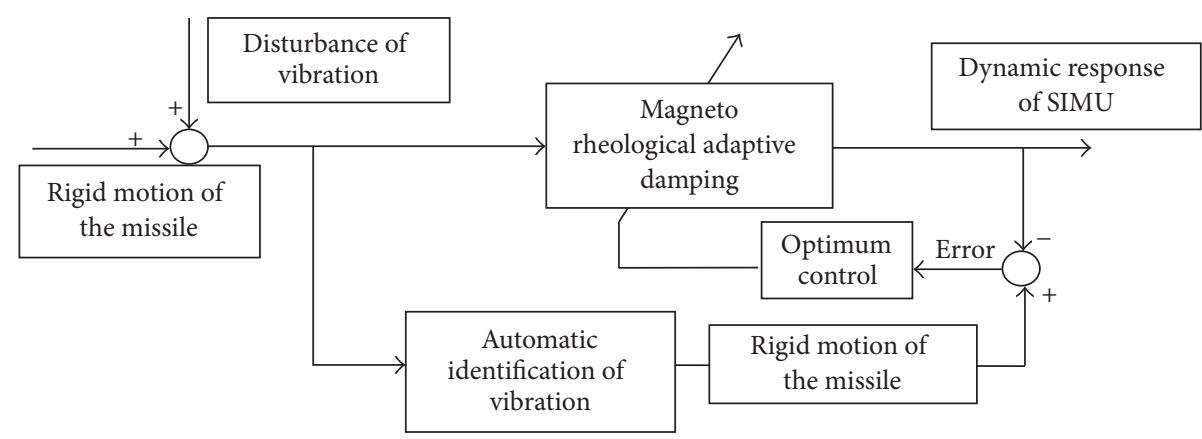

FIgURE 7: The control scheme of adaptive damping system.

\section{The MRE Adaptive Damping System of the Laser Gyro SIMU}

The basic principle of the MRE adaptive damping system [10] of the laser gyro SIMU is that the controller automatically and fast calculates the stiffness and damping parameter of the magneto rheological elastomer that match the dynamic environment according to the variation frequency of the missile during the flight, and the magnetic field intensity in the MRE is changed by controlling the magnitude of current that is transferred to the MRE coil; therefore the stiffness and damping of the MRE are automatically adjusted to a desired value in real time. Therefore, the optimal output control force is produced to eliminate the influence of vibration excitation of the missile, and the dynamic accuracy of SIMU is improved.

In this paper, the rigid motion of the missile is obtained by the technique of automatic determination of the vibration characteristics. The error of the rigid motion of the missile and the dynamic response of SIMU is the performance index of optimal control [11]. The stiffness and damping of the MRE are adjusted automatically by optimal control, the vibration response of SIMU is controlled, and the output accuracy of SIMU is improved. The control scheme of adaptive damping system is shown in Figure 7.

The rigid motion displacement of the missile measured by the SIMU is $x(t)$, the vibration disturbance of missile is $w(t)$, and the dynamic response of SIMU is $y(t)$; then

$$
\dot{y}(t)=\mathbf{A} y(t)+\mathbf{B} F_{c}(t)+\mathbf{H}\left[\begin{array}{c}
x(t)+w(t) \\
\dot{x}(t)+\dot{w}(t)
\end{array}\right] .
$$

That is,

$$
\begin{aligned}
{\left[\begin{array}{l}
y(t) \\
\dot{y}(t)
\end{array}\right]^{\prime}=} & {\left[\begin{array}{cc}
0 & 1 \\
-\frac{k}{m} & -\frac{c}{m}
\end{array}\right]\left[\begin{array}{l}
y(t) \\
\dot{y}(t)
\end{array}\right]+\left[\begin{array}{c}
0 \\
\frac{1}{m}
\end{array}\right] F_{c}(t) } \\
& +\left[\begin{array}{cc}
0 & 1 \\
\frac{k}{m} & \frac{c}{m}
\end{array}\right]\left[\begin{array}{l}
x(t)+w(t) \\
\dot{x}(t)+\dot{w}(t)
\end{array}\right],
\end{aligned}
$$

where $k=k_{\min }, c=c_{\min }, m$ is the mass of SIMU, and $F_{c}(t)$ is the control force. For the measured data of SIMU, the disturbance needs to be eliminated and the rigid motion of the missile needs to be kept. Assuming $e(t)=y(t)-x(t)$, then

$$
\begin{aligned}
{\left[\begin{array}{l}
e(t) \\
\dot{e}(t)
\end{array}\right]^{\prime}=} & {\left[\begin{array}{cc}
0 & 1 \\
-\frac{k}{m} & -\frac{c}{m}
\end{array}\right]\left[\begin{array}{l}
e(t) \\
\dot{e}(t)
\end{array}\right]+\left[\begin{array}{c}
0 \\
\frac{1}{m}
\end{array}\right] F_{c}(t) } \\
& +\left[\begin{array}{cc}
0 & 1 \\
\frac{k}{m} & \frac{c}{m}
\end{array}\right]\left[\begin{array}{l}
w(t) \\
\dot{w}(t)
\end{array}\right] .
\end{aligned}
$$

The objective function of optimal control is

$$
J=\frac{1}{2} \int_{t_{0}}^{t_{f}}\left[\mathbf{e}^{T} \mathbf{Q}_{1} \mathbf{e}+F_{c} \mathbf{Q}_{2} F_{c}\right] d t .
$$

$\mathbf{Q}_{1}(t)$ is the error weighted matrix, $\mathbf{Q}_{2}(t)$ is the control current weighted matrix, $t_{0}$ is the initial time of control, and $t_{f}$ is the end time of control. The linear minimum quadratic Gauss (LQG) control [12] is adopted, according to the separation theorem [13]; then the stochastic optimal control law is

$$
F_{c}(t)=-\mathbf{Q}_{2}^{-1} \mathbf{B}^{T} \mathbf{P e}(t),
$$

where the Riccati algebraic equation that satisfies the controller requirements is

$$
\mathbf{P A}+\mathbf{A} \mathbf{P}^{T}-\mathbf{P B} \mathbf{Q}_{2}^{-1} \mathbf{B}^{T} \mathbf{P}+\mathbf{Q}_{1}=\mathbf{0} .
$$

For the MRE

$$
\begin{aligned}
F_{c}(t)=[ & \left.k(i)-k_{\min }\right][e(t)+w(t)] \\
& +\left[c(i)-c_{\min }\right][\dot{e}(t)+\dot{w}(t)] .
\end{aligned}
$$

According to the mechanics experimental results of the MRE, by fitting, we obtain

$$
\begin{aligned}
& k(i)=k_{\min }+f_{k}(i), \\
& c(i)=c_{\min }+f_{c}(i),
\end{aligned}
$$

where $f_{k}$ is the stiffness function of current and $f_{c}$ is the damping function of current; then

$$
i=\frac{-\mathbf{Q}_{2}^{-1} \mathbf{B}^{T} \mathbf{P e}(t)}{f_{k}^{-1}[e(t)+w(t)]+f_{c}^{-1}[\dot{e}(t)+\dot{w}(t)]},
$$


TABLE 2: The values of stiffness change along three directions of the MRE damper when the current is $0 \mathrm{~A}$ and $2 \mathrm{~A}$, respectively.

\begin{tabular}{lccc}
\hline $\begin{array}{l}\text { Three } \\
\text { directions }\end{array}$ & $\begin{array}{c}\text { Stiffness of the MRE } \\
\text { damper when current is 0 A } \\
(\mathrm{N} / \mathrm{m})\end{array}$ & $\begin{array}{c}\text { Stiffness of the MRE } \\
\text { damper when current is 2 A } \\
(\mathrm{N} / \mathrm{m})\end{array}$ & $\begin{array}{c}\text { Relative variation of } \\
\text { stiffness }(\%)\end{array}$ \\
\hline$X$ direction & $2.85 \times 10^{6}$ & $4.09 \times 10^{6}$ & $43.57 \%$ \\
$Y$ direction & $2.99 \times 10^{6}$ & $4.30 \times 10^{6}$ & $43.97 \%$ \\
$Z$ direction & $2.41 \times 10^{6}$ & $3.51 \times 10^{6}$ & $45.93 \%$ \\
\hline
\end{tabular}

TABLE 3: The values of damping change along three directions of the MRE damper when the current is $0 \mathrm{~A}$ and $2 \mathrm{~A}$, respectively.

\begin{tabular}{lccc}
\hline $\begin{array}{l}\text { Three } \\
\text { directions }\end{array}$ & $\begin{array}{c}\text { Damping of the MRE } \\
\text { damper when current is 0 A } \\
\left(\mathrm{N} \cdot \mathrm{s} \cdot \mathrm{m}^{-1}\right)\end{array}$ & $\begin{array}{c}\text { Damping of the MRE } \\
\text { damper when current is 2 A } \\
\left(\mathrm{N} \cdot \mathrm{s} \cdot \mathrm{m}^{-1}\right)\end{array}$ & $\begin{array}{c}\text { Relative variation of } \\
\text { damping }(\%)\end{array}$ \\
\hline$X$ direction & $8.47 \times 10^{3}$ & $1.28 \times 10^{4}$ & $4.64 \%$ \\
$Y$ direction & $8.89 \times 10^{3}$ & $1.69 \times 10^{4}$ & $43.98 \%$ \\
$Z$ direction & $7.16 \times 10^{3}$ & $1.04 \times 10^{4}$ & $45.25 \%$ \\
\hline
\end{tabular}

where the range of current value is

$$
i=\left\{\begin{array}{l}
2 \\
\frac{-\mathbf{Q}_{2}^{-1} \mathbf{B}^{T} \mathbf{P e}(t)}{f_{k}^{-1}[e(t)+w(t)]+f_{c}^{-1}[\dot{e}(t)+\dot{w}(t)]} \\
0
\end{array}\right.
$$

\section{Experimental Verification}

In order to verify the damping effect of SIMU adaptive system, shaking table experiments of SIMU with the MRE damper and the ordinary rubber shock absorber were carried out, respectively. The scene of the experiments is shown in Figure 8. It is noted that, along each direction, a single MRE device as described in Section 3 was used at each of the four corners of SIMU. Meanwhile, the values of stiffness change along three directions of the adaptive damping system of SIMU are shown in Table 2, and the values of damping change along three directions of the adaptive damping system of SIMU are shown in Table 3. The Bode diagram of the system with the MRE damper and the ordinary rubber shock absorber is shown in Figure 9, respectively. The vibration power spectrum of the missile during the boost phase, the reentry phase, and the whole trajectory is shown in Figure 10. The dynamic response of damping system of SIMU with the MRE damper and the ordinary rubber shock absorber is shown in Figure 11, respectively. It is noted that Figure 9 is a numerical simulation result. Figure 11 is the experimental result of the dynamic response of the SIMU damping system with the MRE damper and ordinary rubber shock absorber, respectively, when the input of the shaking table random vibration test is the vibration power spectrum of the missile measured in the actual navigation test of SIMU. As is seen

$$
\begin{gathered}
\frac{-\mathbf{Q}_{2}^{-1} \mathbf{B}^{T} \mathbf{P e}(t)}{f_{k}^{-1}[e(t)+w(t)]+f_{c}^{-1}[\dot{e}(t)+\dot{w}(t)]} \geq 2 \\
0<\frac{-\mathbf{Q}_{2}^{-1} \mathbf{B}^{T} \mathbf{P e}(t)}{f_{k}^{-1}[e(t)+w(t)]+f_{c}^{-1}[\dot{e}(t)+\dot{w}(t)]}<2 \\
\frac{-\mathbf{Q}_{2}^{-1} \mathbf{B}^{T} \mathbf{P e}(t)}{f_{k}^{-1}[e(t)+w(t)]+f_{c}^{-1}[\dot{e}(t)+\dot{w}(t)]} \leq 0 .
\end{gathered}
$$

from Table 2, the change range of stiffness along three directions is relatively large. As is seen from Table 3, the change range of damping along three directions is relatively large. As is seen from Figure 9, the first-order resonance circular frequency of the system is $242 \mathrm{rad} / \mathrm{s}$ and the amplitude is $14 \mathrm{~dB}$ when the ordinary rubber shock absorber is adopted. Meanwhile, the first-order resonance circular frequency of the system is $268 \mathrm{rad} / \mathrm{s}$ and the amplitude is $-2.41 \mathrm{~dB}$ when the MRE damper is adopted; compared with the ordinary rubber shock absorber, the amplitude is attenuated by $16.41 \mathrm{~dB}$. As is seen from Figure 11, the dynamic response of SIMU is greatly attenuated when the MRE damper and LQG control are adopted. As the magneto rheological force changes rapidly with the magnetic field changes, with almost no delay, the magneto rheological adaptive technology solves the problem that the stiffness of SIMU damping system could not be changed in real time to adapt to the dynamic environment.

\section{Conclusion}

Due to the problem that the output accuracy of SIMU is not high which is in the environment of high overload, strong shock, and large vibration of the missile, the adaptive damping method of MRE as the actuator is designed. The vibration characteristics of SIMU system are fast calculated by establishing the laser gyro SIMU multibody system dynamics 


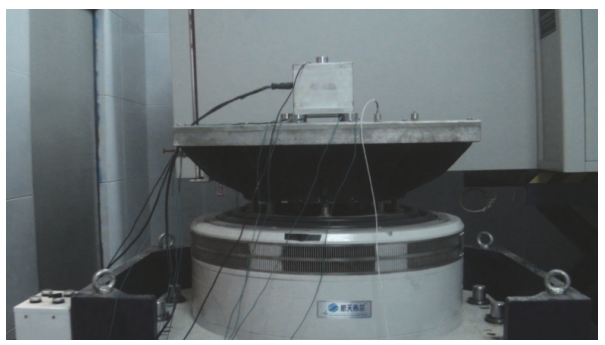

FIGURE 8: The shaking table experiment of SIMU with the MRE damper and the ordinary rubber shock absorber, respectively.

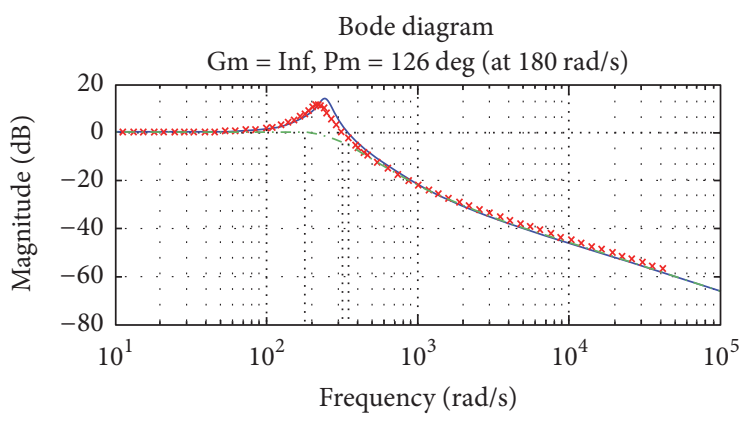

- Ordinary rubber shock absorber

* $\times$ MRE damper with current of $0 \mathrm{~A}$

...- MRE damper with control

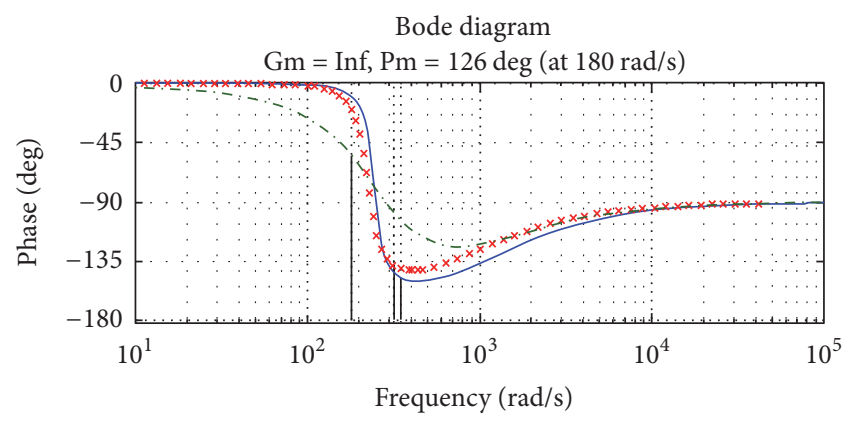

- Ordinary rubber shock absorber

$\times \quad \times$ MRE damper with current of $0 \mathrm{~A}$

- - MRE damper with control

FIGURE 9: The Bode diagram of the damping system with the MRE damper and the ordinary rubber shock absorber, respectively.

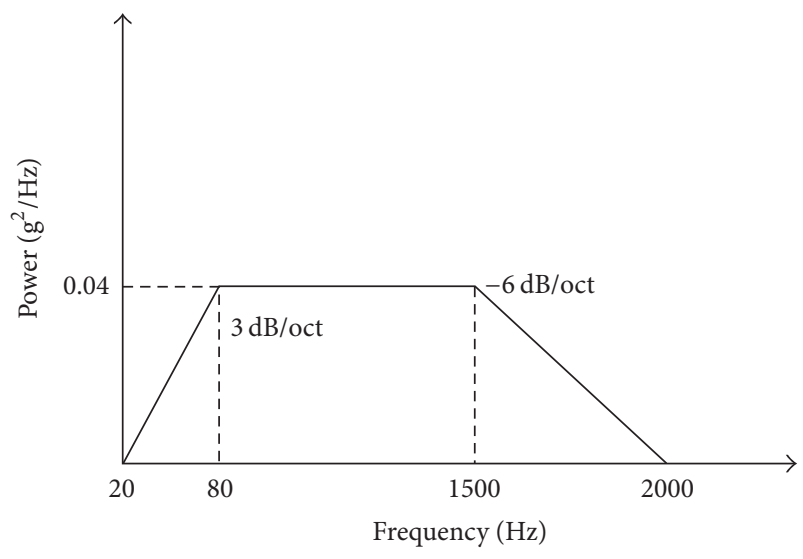

(a) The vibration power spectrum of the missile during the boost phase

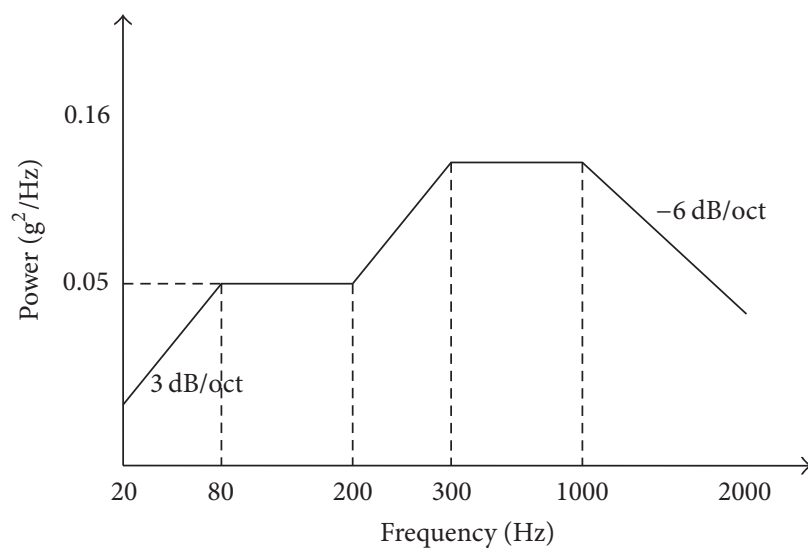

(b) The vibration power spectrum of the missile during the reentry phase

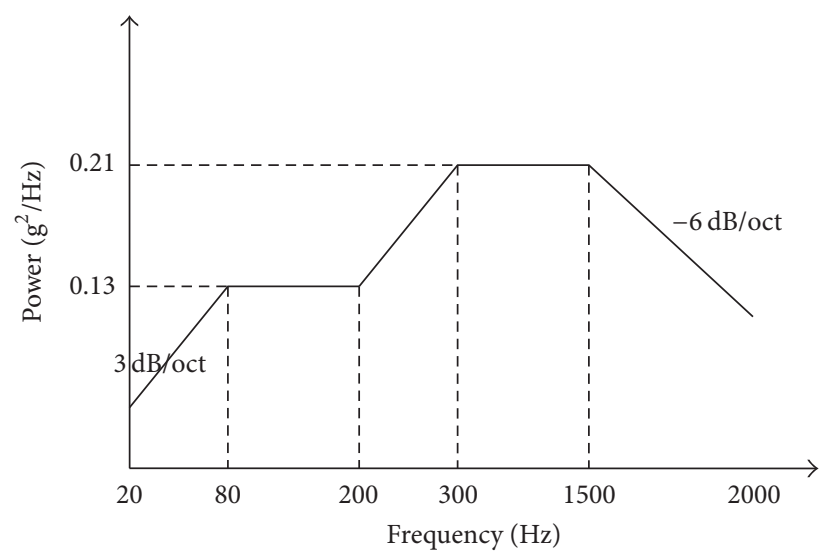

(c) The vibration power spectrum of the missile during the whole trajectory

FIGURE 10: The vibration power spectrum of the missile during the boost phase, the reentry phase, and the whole trajectory. 


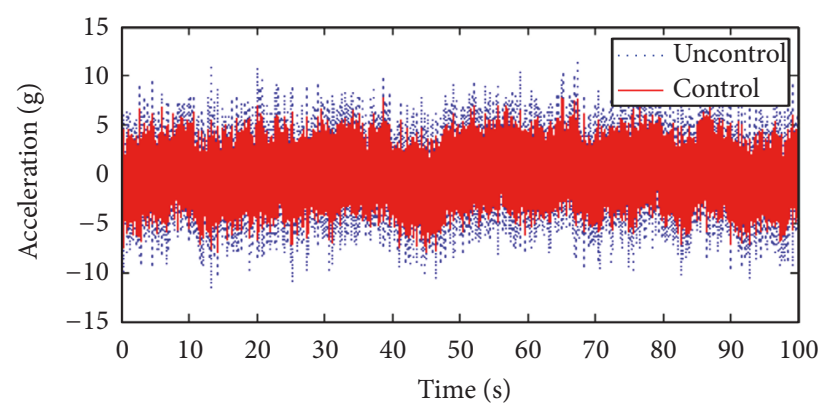

(a) The boost phase of the missile flight

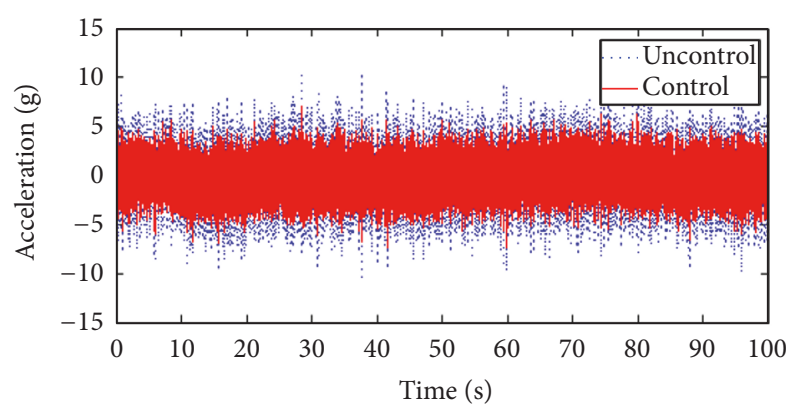

(b) The reentry phase of the missile flight

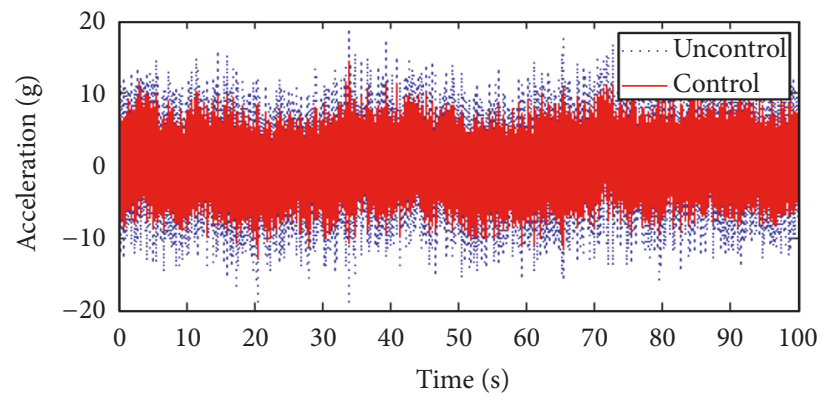

(c) The whole trajectory of the missile flight

FIGURE 11: The dynamic response of SIMU damping system with the MRE damper and the ordinary rubber shock absorber, respectively.

model and getting the transfer equation and transfer matrix of each element. Then the vibration response of the missile is calculated by the automatic determination technique of vibration characteristics. The stiffness and damping of the MRE are adjusted automatically by optimal control, and the vibration response of SIMU is controlled. Therefore, the output accuracy of SIMU is improved. The results show that the fast calculation method of vibration characteristics of SIMU is very efficient, and the design of adaptive damping system also achieves the desired effect for SIMU; meanwhile, this solves the problem that the stiffness and damping of SIMU could not be changed in real time to adapt to the dynamic environment.

\section{Conflicts of Interest}

The authors declare that there are no conflicts of interest regarding the publication of this paper.

\section{References}

[1] J. M. Ginder, W. F. Schlotter, and M. E. Nichols, "Magnetorheological elastomers in tunable vibration absorbers," Proceedings of SPIE-The International Society for Optical Engineering, vol. 4331, pp. 103-110, 2001.

[2] A. Joshi, "Free vibration characteristics of variable mass rockets having large axial thrust/acceleration," Journal of Sound and Vibration, vol. 187, no. 4, pp. 727-736, 1995.

[3] R. Xiaoting, Transfer Matrix Method of Multibody System and Its Application, Science Press, 2008.

[4] R. Bao, Study on Transfer Matrix Method for Dynamics of Controlled Multibody Systems, Dissertation for the Doctoral
Degree in Engineering of Nanjing University of Science and Technology, 2011.

[5] G. Chen, X. Rui, F. Yang, J. Zhang, and Q. Zhou, "Study on the dynamics of laser Gyro strapdown inertial measurement unit system based on transfer matrix method for multibody system," Advances in Mechanical Engineering, vol. 2013, Article ID 854583, 2013.

[6] A. V. Panishev, "The dichotomy method in the three-machine problem," Soviet J. comput. systems Sci, vol. 1, pp. 181-187, 1987.

[7] D. I. J. Wittenburg, Dynamics of Systems of Rigid Bodies, Teubner, 1977.

[8] F. Alizadeh, "Interior point methods in semidefinite programming with applications to combinatorial optimization," SIAM Journal on Optimization, vol. 5, no. 1, pp. 13-51, 1995.

[9] W. Blajer, D. Bestle, and W. Schiehlen, "An orthogonal complement matrix formulation for constrained multibody systems," Journal of Mechanical Design, vol. 116, no. 2, pp. 423-428, 1994.

[10] Y. Shen, M. F. Golnaraghi, and G. R. Heppler, "Experimental research and modeling of magnetorheological elastomers," Journal of Intelligent Material Systems and Structures, vol. 15, no. 1, pp. 27-35, 2004.

[11] A. Rantzer and M. Johansson, "Piecewise linear quadratic optimal control," Institute of Electrical and Electronics Engineers. Transactions on Automatic Control, vol. 45, no. 4, pp. 629-637, 2000.

[12] D. S. Bernstein and M. M. Haddad, "LQG control with an Ho, performance bound: a Riccati equation approach," Automatic Control IEEE Transactions, vol. 34, no. 3, pp. 293-305, 1989.

[13] N. Halyo and A. K. Caglayan, "A separation theorem for the stochastic sampled-data LQG problem," International Journal of Control, vol. 23, no. 2, pp. 237-244, 1976. 


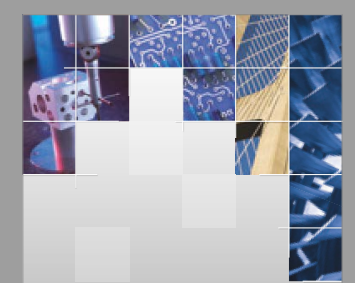

\section{Enfincering}
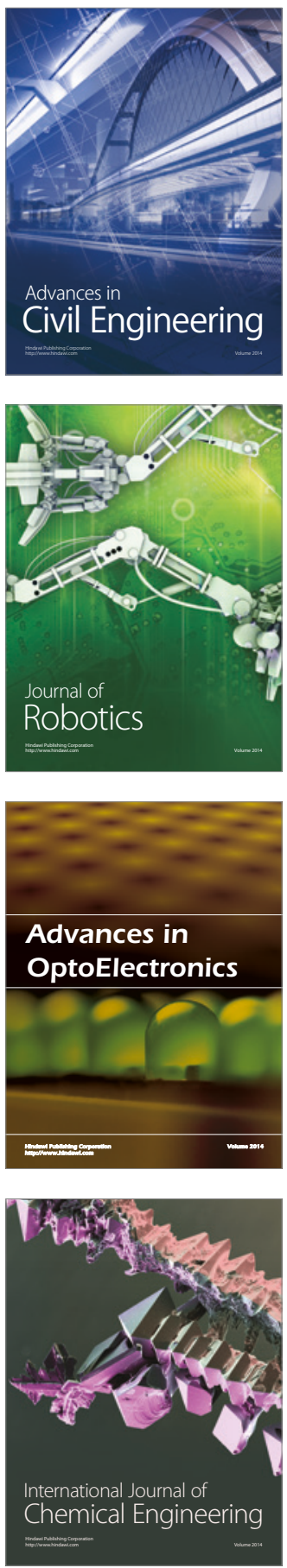

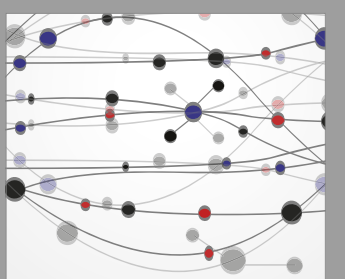

The Scientific World Journal

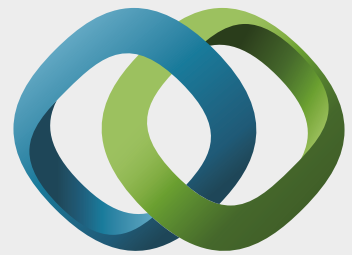

\section{Hindawi}

Submit your manuscripts at

https://www.hindawi.com
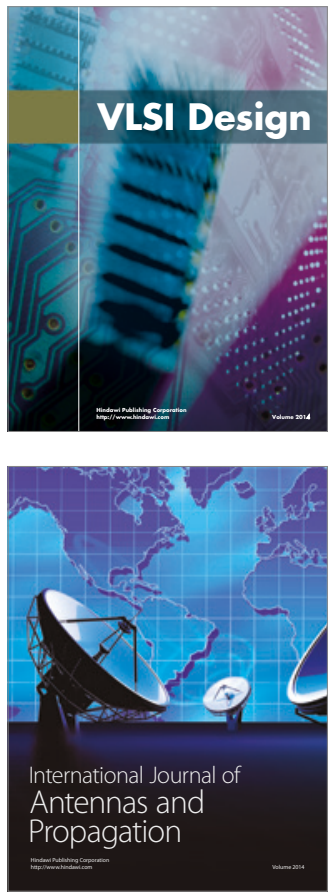

\section{Rotating}

Machinery
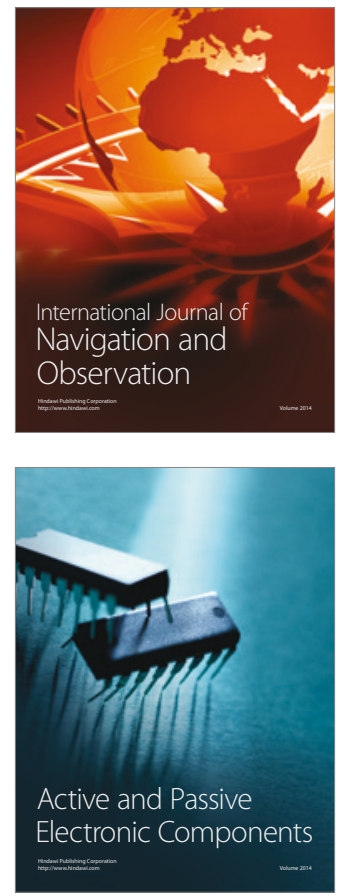
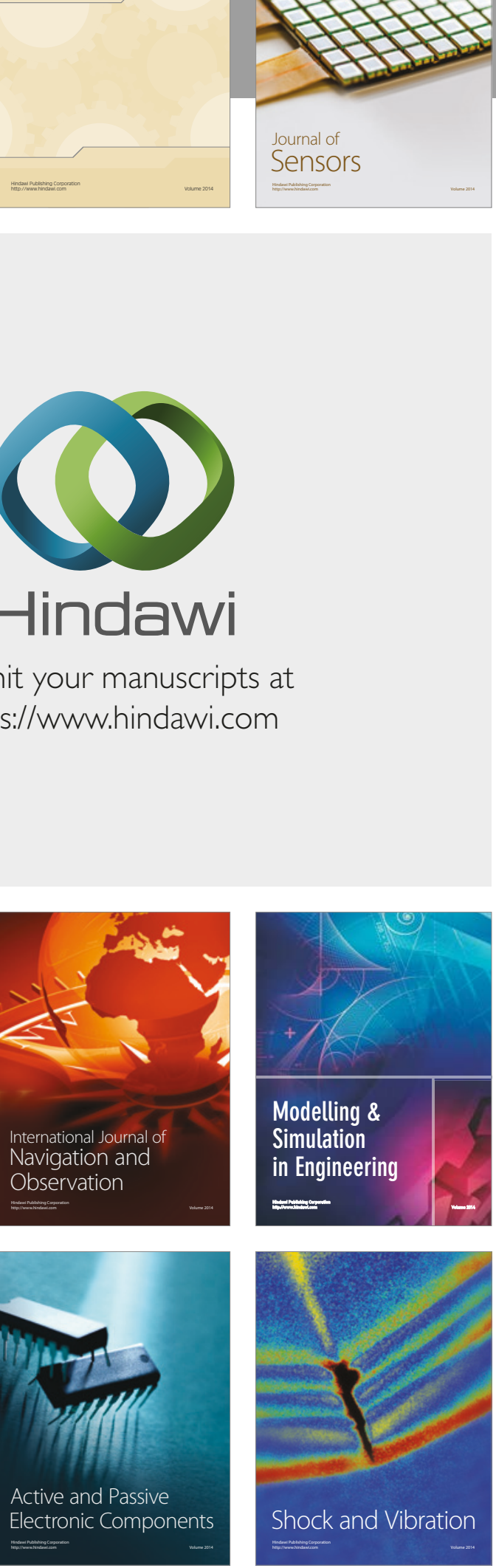
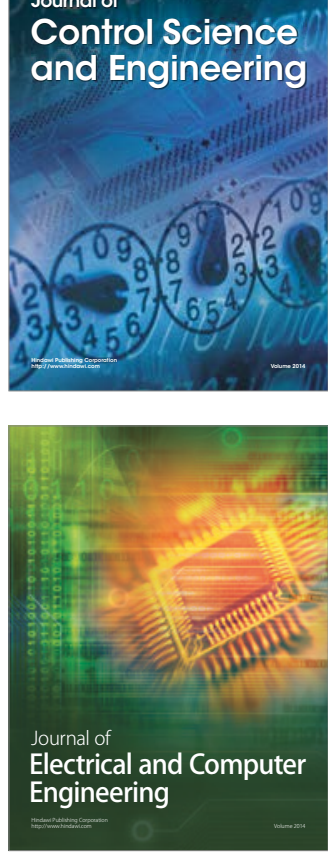

Distributed

Journal of

Control Science

and Engineering
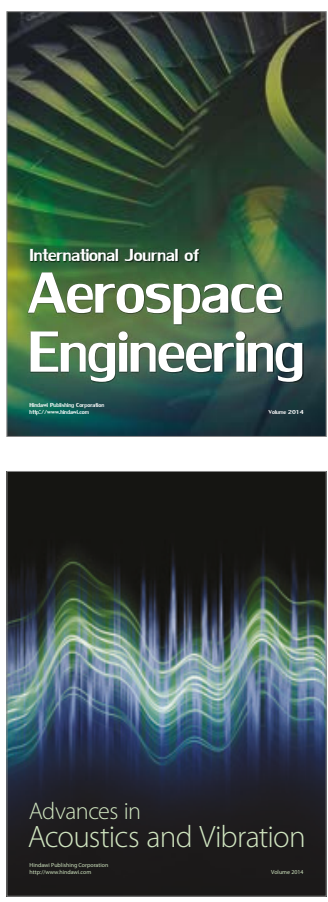

Sensor Networks 